\title{
Extracorporeal Membrane Oxygenation: The First 10 Years Experience of a Portuguese Pediatric Intensive Care Unit
}

\author{
Extracorporeal Membrane Oxygenation: Os Primeiros \\ 10 Anos de Experiência de uma Unidade de Cuidados \\ Intensivos Pediátricos Portuguesa
}

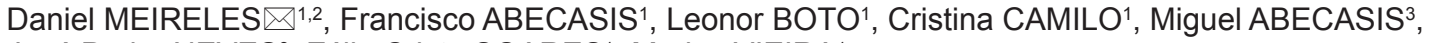 \\ José Pedro NEVES ${ }^{3}$, Zélia Cristo SOARES ${ }^{1}$, Marisa VIEIRA ${ }^{1}$
}

Acta Med Port 2021 Jun;34(6):435-441 - https://doi.org/10.20344/amp.15227

\section{ABSTRACT}

Introduction: In Portugal, extracorporeal membrane oxygenation (ECMO) is used in pediatric patients since 2010. The aim of this study was to describe the clinical characteristics of patients, indications, complications and mortality associated with the use of ECMO during the first 10-years of experience in the Pediatric Intensive Care Unit located in Centro Hospitalar Universitário Lisboa Norte.

Material and Methods: Retrospective observational cohort study of all patients supported with ECMO in a Pediatric Intensive Care Unit, from the $1^{\text {st }}$ of May 2010 up to $31^{\text {st }}$ December 2019.

Results: Sixty-five patients were included: 37 neonatal ( $\leq 28$ days of age) and 28 pediatric patients ( $>28$ days). In neonatal cases, congenital diaphragmatic hernia was the main reason for ECMO ( $40 \%$ of neonatal patients and $23 \%$ of total). Among pediatric patients respiratory distress was the leading indication for ECMO (47\% of total). The median length of ECMO support was 12 days. Clinical complications were more frequent than mechanical complications (65\% vs 35\%). Among clinical complications, access site bleeding was the most prevalent with $38 \%$ of cases. The overall patient survival was $68 \%$ at the time of discharge $(65 \%$ for neonatal and $71 \%$ for pediatric cases), while the overall survival rate in Extracorporeal Life Support Organization registry was $61 \%$. The number of ECMO runs has been increasing since 2011, even though in a non-linear way (three cases in 2010 to 11 cases in 2019).

Discussion: In the first 10 years we received patients from all over the country. Despite continuous technological developments, circuitrelated complications have a significant impact. The overall survival rate in the Pediatric Intensive Care Unit was not inferior to the one reported by the Extracorporeal Life Support Organization.

Conclusion: The overall survival of our Pediatric Intensive Care Unit is not inferior to one reported by other international centers. Our experience showed the efficacy of the ECMO technique in a Portuguese centre.

Keywords: Extracorporeal Membrane Oxygenation; Intensive Care Units, Pediatric; Portugal

\section{RESUMO}

Introdução: Em Portugal, a oxigenação por membrana extracorporal (ECMO) é utilizado em doentes pediátricos de forma consistente desde 2010. O nosso objetivo é descrever as características clínicas, indicações, complicações e sobrevivência associadas à utilização da ECMO nos primeiros 10 anos de experiência na nossa unidade.

Material e Métodos: Estudo de coorte retrospetivo dos doentes tratados com ECMO na Unidade de Cuidados Intensivos Pediátricos do Hospital de Santa Maria, de 1 de maio de 2010 a 31 de dezembro de 2019

Resultados: Foram incluídos 65 doentes: 37 neonatais ( $\leq 28$ dias de idade) e 28 pediátricos ( $>28$ dias). Nos neonatais, a hérnia diafragmática congénita foi a principal indicação ( $40 \%$ dos recém-nascidos e $23 \%$ do total). Relativamente aos doentes pediátricos, a insuficiência respiratória constituiu a principal indicação para ECMO (47\% do total). A mediana de duração da técnica foi de 12 dias As complicações clínicas foram mais frequentes do que as mecânicas (65\% vs $35 \%$ ). Entre as complicações clínicas, a hemorragia no local de acesso foi a mais frequente (38\% dos casos). A sobrevivência global do total da amostra foi de $68 \%$ no momento da alta (65\% nos neonatais e $71 \%$ nos pediátricos), enquanto que a sobrevivência descrita no registo da Extracorporeal Life Support Organization é de $61 \%$. O número de casos de utilização de ECMO tem vindo a aumentar desde 2011, embora de forma não linear (três casos em 2010 para 11 casos em 2019).

Discussão: Nos primeiros 10 anos de experiência em ECMO na Unidade de Cuidados Intensivos Pediátricos recebemos doentes oriundos de várias partes do país. Apesar da evolução tecnológica contínua, as complicações relacionadas com o circuito têm um impacto significativo. A taxa de sobrevida global no nosso centro não foi inferior à reportada no relatório da Extracorporeal Life Support Organization.

Conclusão: A sobrevida global na Unidade de Cuidados Intensivos Pediátricos não é inferior à descrita no registo internacional. A nossa experiência demonstra a eficácia da ECMO num centro Português.

Palavras-chave: Cuidados Intensivos Pediátricos; Oxigenação por Membrana Extracorporal; Portugal

\section{INTRODUCTION}

Extracorporeal membrane oxygenation (ECMO) is a technique of cardiopulmonary support that provides respiratory and/ or hemodynamic assistance in critical ill patients.

The terms ECMO and extracorporeal life support (ECLS) are used frequently as synonyms that mean temporary support of pulmonary or cardiac function using mechanical

1. Unidade de Cuidados Intensivos Pediátricos. Departamento de Pediatria. Hospital de Santa Maria. Centro Hospitalar Universitário de Lisboa Norte. Lisboa. Portugal.

2. Serviço de Pediatria. Centro Materno-Infantil do Norte. Centro Hospitalar Universitário do Porto. Porto. Portugal.

3. Serviço de Cirurgia Cardiotorácica. Hospital de Santa Cruz. Centro Hospitalar de Lisboa Ocidental. Lisboa. Portugal.

$\triangle$ Autor correspondente: Daniel Meireles. daniel.r.meireles@gmail.com

Recebido: 03 de novembro de 2020 - Aceite: 20 de abril de 2021 - Online issue published: 01 de junho de 2021

Copyright $\odot$ Ordem dos Médicos 2021 
devices. ${ }^{1}$ By definition, ECLS includes several types of life support techniques, including ECMO.

The beginning of ECLS can be dated back to the 1950s when John Gibbon, an American surgeon, built the first heart-lung machine.

Robert Bartlett, an American cardiothoracic surgeon, successfully treated the first infant with ECMO, a little girl named Esperanza in $1975 .^{2}$ Ten years later he presented the case series of neonates treated with ECMO. This study included 100 patients and revealed that the quality of survival is the same or better than that reported for conventional respiratory care. ${ }^{3}$

In 1989, the Extracorporeal Life Support Organization (ELSO) was created with the aim to organize and spread the knowledge about ECMO and, therefore to increase the use of this technique. ${ }^{2}$

The global use of ECMO in every ECMO center is registered in ELSO and, twice a year, there is an activity report. According to ELSO (January 2020), approximately 130000 ECMO runs were reported worldwide at more than 300 centers from 1990 to 2019.4

The ECMO technique not only improves clinical outcomes, but it is also cost effective in adults with severe respiratory failure when compared with conventional ventilation support. 5

As mentioned above, the first neonatal ECMO case was described more than 40 years ago. ${ }^{6}$ However, in Portugal, ECMO was only used for the first time, outside the context of cardiac surgery, in May 2010, in our institution. Centro Hospitalar Universitário Lisboa Norte (CHULN) started the ECMO program in 2010 in the context of the Influenza A $\mathrm{H} 1 \mathrm{~N} 1$ pandemic. Driven by the adult intensive care unit (ICU) decision to start an ECMO program, our pediatric ICU embraced the challenge and developed the neonatal and pediatric ECMO center. Soon after setting up the program, we had the first case in May 2010. The program was based on protocols developed by the neonatal and pediatric intensive care units and on the support of cardiothoracic surgeons and perfusionists. Although the first case allowed us to understand that we were capable of managing ECMO patients, it became clear that we needed to receive more training from established ECMO centers. During the summer of 2010 two doctors and a nurse from our pediatric intensive care unit (PICU) went to the Karolinska Institute in Stockholm to learn in one of the best ECMO centers in the world. Soon after, a formal protocol was established with the cardiothoracic surgery department of Centro Hospitalar Lisboa Ocidental to ensure the collaboration of pediatric cardiothoracic surgeons in the cannulation and decannulation process. Formal training sessions with hands-on workshops were also implemented and are carried out twice a year for all PICU staff. Since 2010, two hospitals in Portugal have been treating pediatric patients with ECMO: Hospital de São João (Porto) and Hospital de Santa Maria (Centro Hospitalar Universitário Lisboa Norte). The first case series of both hospitals were reported a few years later. ${ }^{7,8}$

In 2017, the ECMO program of CHULN was recognized as a national referral center. It includes three sections: respiratory adult venovenous ECMO (VV-ECMO), cardiac adult venoarterial ECMO (VA-ECMO) and the neonatal and pediatric ECMO (both VV and VA).

The aims of this study were to review all patients treated with ECMO in the PICU of Centro Hospitalar Universitário Lisboa Norte, to understand the impact of the implementation of this technique in the population studied and to compare our results with those of the largest international registry of ECMO patients.

\section{MATERIAL AND METHODS Data collection}

We performed an observational, retrospective analysis from a prospectively collected database. Data were obtained from our institutional electronic medical records of neonatal and pediatric patients who required ECMO. All patients treated with ECMO from May 2010 to December 2019 in the PICU of our ECMO center were included in the study. The criteria for ECMO were based on current indications as described below, and all cases were discussed with the Pediatric ECMO coordinator (FA) before acceptance.

The Ethics Committee of Centro Hospitalar Universitário Lisboa Norte, Faculdade de Medicina de Lisboa, approved the implementation and publication of this study and waived the need for informed consent.

Our analysis included: demographic characteristics (age and gender), indications for ECMO, mode, duration, complications and outcome (survival at 24 hours after decannulation and the time of discharge). We considered survival if the patients were alive at the time of discharge or were transferred to another hospital. The ELSO registry defines Neonatal ECMO patients as less than or equal to 28 days of age at the time of cannulation and Pediatric patients as those patients 29 days to 17 years of age at the time of cannulation.

\section{Definitions and concepts \\ Indications and contraindications}

There are two types of ECMO: venoarterial (VA) and venovenous (VV). The first one is used for heart and lung support - the blood is taken out of a large vein, passes through the silicon heparin- coated tubes to the centrifugal pump, then through the polymethylpentene hollow fiber membrane oxygenator where gas exchange takes place, and returns oxygenated to the arterial system. The VV ECMO is used for lung support only - two venous cannulas are introduced in the venous system, the blood is pulled out from one vein to the ECMO circuit and returns back oxygenated to the venous system in spots close to the heart.

The neonatal patients eligible for ECMO can be divided in two large groups: primary condition associated with pulmonary hypertension (such as idiopathic primary pulmonary hypertension of the newborn, respiratory distress syndrome, meconium aspiration syndrome, sepsis and asphyxia) and congenital diaphragmatic hernia patients. ${ }^{9}$

The indications for ECMO in pediatric patients vary 
Table 1 - Description of the population of our study by age group $(n=65)$

\begin{tabular}{lccc}
\hline Outcome measures & Neonatal & Pediatric & Total \\
\hline Total $(\mathrm{n}, \%)$ & $37(57 \%)$ & $28(43 \%)$ & 65 \\
Gender (F / M) & $51 \% / 49 \%$ & $43 \% / 57 \%$ & $48 \% / 52 \%$ \\
Age & & & $15 \mathrm{~d}(1 \mathrm{~d}-17 \mathrm{y})$ \\
median (min. - max.) & $3 \mathrm{~d}(0-20 \mathrm{~d})$ & $32 \mathrm{~m}(29 \mathrm{~d}-17 \mathrm{y})$ & $2-416 \mathrm{~d}$ \\
IQR & $1-7 \mathrm{~d}$ & $5-131 \mathrm{~m}$ & $12 \mathrm{~d}(1-57 \mathrm{~d})$ \\
Length of support (median, min. - max.) & $12 \mathrm{~d}(1-57 \mathrm{~d})$ & $13 \mathrm{~d}(1-34 \mathrm{~d})$ & 47 \\
ECMO mode & & & 11 \\
V-A & 36 & 17 & 18 \\
V-V & 1 & $20(69 \%)$ & $47(71 \%)$ \\
Survival at the time of decannulation $(\mathrm{n}, \%)$ & $28(76 \%)$ & $20(69 \%)$ & $44(68 \%)$ \\
Survival at the time of discharge $(\mathrm{n}, \%)$ & $24(65 \%)$ & & 47 \\
\hline
\end{tabular}

F: female; M: male; d: day; y: years; m: months; V-A: veno-arterial; V-V: veno-venous

among different authors, but common criteria include, for VV ECMO: respiratory failure (severe acute hypoxemic respiratory failure with oxygenation index over 40 for more than three hours with aggressive conventional ventilation or severe acute hypercapnic respiratory failure with $\mathrm{PaCO}_{2}$ over $100 \mathrm{mmHg}$ for more than six hours, despite aggressive conventional ventilation). The criteria for VA ECMO include sepsis with severe distributive shock (after the first six hours of goal directed resuscitation), severe cardiac dysfunction with signs of hypoperfusion (after adequate volume resuscitation and inotropic/ vasopressor support) such as cardiogenic shock and myocarditis and cardiomyopathies, refractory cardiopulmonary resuscitation (CPR), bridge to assist device or heart transplant. ${ }^{10}$ In fact, congenital heart disease $(\mathrm{CHD})$ is also an important diagnosis for neonates and children supported with ECMO - VA ECMO is used in cases of heart failure after CHD surgery and in post-cardiotomy to allow myocardial recovery in bridge patients or to provide support as a bridge to cardiac transplantation.

According to ELSO, ECMO should be considered if the mortality risk is $50 \%$ and is indicated if the mortality risk is $80 \%$, despite maximum medical treatment.

Contraindications for ECMO are often known irreversible conditions or associated with a poor outcome. These conditions include neurological injuries, intracranial hemorrhage, irreversible multiorgan failure, untreatable malignancy, unrecoverable severe heart disease and prolonged arrest time. A relative contraindication for ECMO use is a prolonged mechanical ventilation with barotrauma. ${ }^{1}$ In neonates, the main limitations are related to the weight (under $2 \mathrm{~kg}$ ), preterm delivery (under 35 weeks of gestational age) and the need for systemic anticoagulation. ${ }^{7}$

\section{Complications}

Complications occur frequently. They can be classified as disease complications or technique complications. The latter include hemorrhage, limb ischemia, stroke, thrombosis and infection. Some studies in adult populations showed that at least one significant complication occurs in over half of patients on ECMO, with bleeding as the most frequent complication. ${ }^{1}$

Both types of ECMO (VV-ECMO and VA-ECMO) can lead to complications. Long duration of ECMO, usually called long runs, is probably the most important risk factor for developing healthcare-associated infections (such as respiratory, bloodstream and urinary tract infections). ${ }^{11}$

In addition to local infection in the cannula site, vascular complications such as thrombosis, major bleeding and ischemia are frequent, mainly with VA-ECMO, as is described in studies in the adult population. ${ }^{12}$

The main causes of morbidity and mortality in ECMO patients are thrombosis and bleeding. ${ }^{13}$ Neurological complications are also an important factor to consider and contribute to the overall mortality rate of children in ECMO. Intracranial hemorrhage $(\mathrm{ICH})$ is frequent among these patients and the incidence of $\mathrm{ICH}$ is higher in children when compared to adults. ${ }^{14,15}$ In neonatal patients some studies like the one by Thiagarajan et al. demonstrated that preECMO factors as prematurity, lower body weight, cardiac arrest, and physiological instability increased the chances of neurological complications. ${ }^{15}$

\section{Follow-up}

All patients that underwent ECMO are evaluated in the outpatient clinic after discharge by one of the PICU physicians. The periodicity varies between 3 to 6 months according to age and clinical status. All neonatal patients are also evaluated in the neurodevelopmental clinic with formal evaluation of development.

\section{Statistical analysis}

Continuous variables are expressed as medians and interquartile ranges and categorical variables as absolute values and percentage. Statistical analysis was performed using Student t-test or Mann-Whitney-U test, with each depending on whether continuous variables were normally distributed or not and the Chi-square test for categorical variables. Statistical significance was defined as $p<0.05$. All statistical analysis was performed by using SPSS Version 25.0 (IBM Corp, Chicago, IL, USA). 


\section{RESULTS}

Since ECMO was implemented in our PICU, this technique was required for a total of 65 patients: 37 neonatal patients $(57 \%)$ and 28 pediatric patients (43\%). In these patients, we performed a total of 67 ECMO runs (two patients one neonatal and one pediatric - had been on ECMO twice).

Among neonatal patients the median age was three days (min. 0; max. 20 days, interquartile range - IQR: 1-7 days). The median age of pediatric patients was three years and six months (min. 29 days; max. 17 years and four months, IQR: 5 - 131 months). Fifty two percent of patients were male. Table 1 describes population demographics.

Fifty four percent of patients $(n=35)$ included in this study came from other hospitals in different regions in
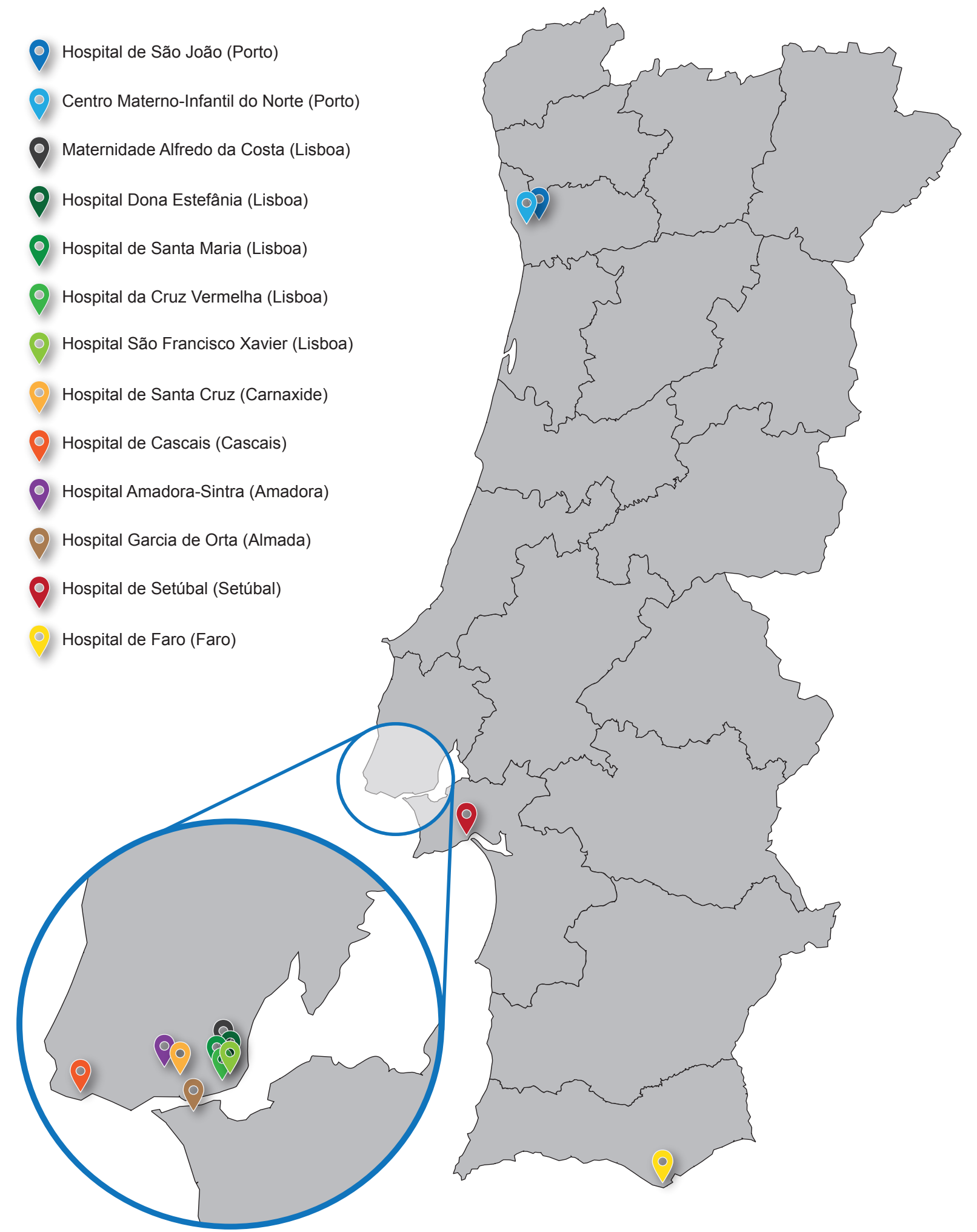
Portugal (Fig. 1). Hospital Professor Dr. Fernando Fonseca ( $n=10,15 \%$ ), Hospital Dona Estefânia (Centro Hospitalar Lisboa Central, $n=7,11 \%$ ) and Hospital Garcia da Orta ( $\mathrm{n}$ $=6,9 \%$ ) were the main referring hospitals.

The main indication for ECMO was respiratory disease (47\%, including asthma, pneumonia, bronchiolitis, pulmonary hypertension), followed by congenital diaphragmatic hernia $(\mathrm{CDH}, 23 \%$ of total). Among the neonatal patients, $\mathrm{CDH}$ was the most frequent indication for ECMO $(n=15$, $40 \%$ of neonatal cases) followed by meconium aspiration syndrome $(n=6,16 \%)$. Among the pediatric population, we performed ECMO in 11 cases for pneumonia (39\% of pediatric cases). Bronchiolitis was the second cause for ECMO use in pediatric cases $(n=4,14 \%)$. In our database, the underlying cardiac disease for ECMO eligibility was present in three patients: two neonatal (one for total anomalous pulmonary venous return and the other for double outlet right ventricle) and one pediatric (myocarditis).

Overall, venoarterial (VA) ECMO was used in $72 \%$ of cases: of the 28 pediatric patients, 11 were placed on VA ECMO and 17 on VV ECMO; of the 37 neonatal cases, 36 were placed on VA and a single one was run on VV ECMO.

The median length of ECMO support was 12 days (min. 1; max. 57 days; IQR: 7.5 - 19 days). The conditions related to the longest ECMO duration were surfactant deficiency (57 days), pulmonary aspergillosis (34 days), pneumonia in a patient with rhabdomyosarcoma (26 days) and in a patient with cystic fibrosis ( 25 days).

Complications occurred 161 times in our population. One hundred and four complications were described in neonatal patients $(65 \%)$ and 57 times in the pediatric population (35\%).

There were two types of technical complications: mechanical complications related with the procedure $(n=56$, $35 \%$ ) and clinical complications ( $n=105,65 \%)$. Considering the mechanical complications, the circuit component clots was the most frequent $(n=32,57 \%)$ followed by cannula problems $(n=15,27 \%)$. The hemorrhagic complications

Table 2 - Total ECMO runs since 2010 in UCIPed

\begin{tabular}{lccc}
\hline \multicolumn{1}{c}{ Time period } & Neonatal & Pediatric & Total \\
\hline $\mathbf{2 0 1 0 - 2 0 1 4}$ (5 years) & $\mathbf{1 5}$ & $\mathbf{1 0}$ & $\mathbf{2 5}$ \\
2010 & 0 & 3 & 3 \\
2011 & 1 & 1 & 2 \\
2012 & 3 & 2 & 5 \\
2013 & 5 & 2 & 7 \\
2014 & 6 & 2 & 8 \\
$2015-2019$ (5 years) & $\mathbf{2 3}$ & $\mathbf{1 9}$ & $\mathbf{4 2}$ \\
2015 & 3 & 2 & 5 \\
2016 & 4 & 7 & 11 \\
2017 & 6 & 1 & 7 \\
2018 & 3 & 5 & 8 \\
2019 & 7 & 4 & 11 \\
\hline
\end{tabular}

UCIPed: unidade de Cuidados Intensivos Pediátricos were the most prevalent among the clinical complications ( $n=48,46 \%)$. Cannulation site bleeding $(n=18,38 \%)$, and surgical site bleeding ( $n=12,25 \%$, corresponding to 11 cases of $\mathrm{CDH}$ repair and one case of pheochromocytoma excision) were the main hemorrhagic complications. In our population there were seven cases of gastrointestinal hemorrhage $(15 \%)$ and in six cases there was disseminated intravascular coagulation (13\%). Neurological complications were described 19 times (18\% of clinical complications); central nervous system (CNS) infarction and CNS hemorrhage being the most frequent ( 9 and 5 cases respectively, $47 \%$ and $26 \%$ ).

Survival at the time of discharge was $68 \%(n=44)$.

The outcome (survival/ non-survival) was not associated with age $(p>0.05)$. On the other hand, we found an association between gender and outcome $(p=0.019)$, with a higher mortality observed in male gender (47\% vs $19 \%)$.

When we considered two groups depending on ECMO run duration ( $\leq 7$ days and $>7$ days), the results demonstrated an association between longer ECMO duration and survival $(p=0.03)$. An ECMO duration over 7 days was associated with lower mortality ( $25 \%$ of deaths vs $56 \%$ for $\leq 7$ days duration).

All patients had regular follow-up at the outpatient clinic carried out by one of the PICU doctors. In terms of clinical outcomes, we found that three cases $(5 \%)$ had severe neurologic sequelae: two neonatal and one pediatric patient. In these cases, the median duration of ECMO was 10 days. In one case, ECMO was used as a bridge to lung transplantation and another patient was diagnosed with autism during follow-up. None of all other patients had any significant sequelae so far.

The number of patients treated with ECMO has been increasing: in 2010 we performed three cases, in 2019, 11 cases. The trend towards an increase in the number of ECMO runs during the study period is shown in Table 2.

\section{DISCUSSION}

According to the ELSO Guidelines, ECLS centers should be located in geographic locations that have a minimum of six patients per year. ${ }^{16}$ Our unit has received patients from all over the country and has been a referral center for neonatal and pediatric ECMO treatment over the last 10 years. More frequently, our cases are patients from other hospitals ( $n=34,54 \%$ ). In the last eight years, the number of ECMO runs per year ranged from 7 - 11 cases, with the exception for 2015 ( $n=5$ cases). This is in agreement with ELSO guidelines for maintaining an ECMO program.

No significant sociodemographic differences were observed between age (neonatal and pediatric) or gender groups in our population. The most frequent diagnosis leading to ECMO support were respiratory conditions and $\mathrm{CDH}$, which is consistent with the ELSO registry. The cardiac causes such as post-cardiotomy shock are a common reason for ECMO, generally with a less favorable outcome. But in our data we had only three cases of cardiac disease $(5 \%)$ as mentioned above, which is not meaningful. 
This happens because of the organization of the care of pediatric patients with cardiac disease. The two public hospitals that have pediatric cardiac surgery are located in hospitals that do not have maternity or pediatric departments. When these patients need post-cardiotomy ECMO it is carried out in the hospitals where they are operated. This is a different kind of ECMO that has been available for many years and is beyond the scope of this paper.

Despite the evolution, improvement, and innovation in ECMO equipment, circuit-related complications continue to have an important impact on these patients. According to the ELSO Registry International Report, ${ }^{16}$ thrombus and cannula problems are the two most frequent mechanical complications in ECMO patients, which is similar to our results.

The most common clinical complication in our cases was hemorrhagic complications such as cannulation site bleeding (39\%) and the surgical site bleeding (25\%). Some studies reported an overall CNS complication rate of $10.9 \%$ - $12.9 \% .{ }^{12,14,17}$ Our population had an overall CNS complication of $12 \%$ and the most frequent complication was CNS infarction $(47 \%$ of total neurologic problems described in our patients).

When compared to the ELSO registry, overall survival in our institution for neonatal and pediatric patients was not lower (68\% versus $61 \%)$. In this review we could not find any differences in terms of survival between the different age groups analyzed (neonates and pediatric patients), which is different from other studies described in the literature and demonstrates that younger age was associated with higher mortality. ${ }^{18}$ A study revealed that survival was inversely correlated with ECMO duration, particularly in cases of support for more than seven days. That was probably the cause because prolonged duration of ECMO use is associated with more severe diseases. ${ }^{19}$ Curiously, we found an association between ECMO duration and survival $(p=0.03)$, with higher mortality in ECMO duration group under 7 days (50\%) when compared to ECMO duration over seven days (18\%). This can probably be explained by the patients with $\mathrm{CDH}$ who died shortly after surgery, but also by the patient's poor prognosis condition at PICU admission: two patients with whooping cough, three post bone marrow transplant and two with severe sepsis.

ECMO is also associated with morbidity. Quality of life is an extremely important parameter for the evaluation of the outcomes of these patients. In our study we found major neurologic sequelae in $5 \%$ of survival cases $(n=3)$, which is lower compared to the findings of some published studies. ${ }^{20}$ Our ECMO patients have regular follow-up after discharge to evaluate the clinical outcomes. An unpublished study performed at our center (Morais L, unplished master's degree thesis, University of Lisbon; 2016) characterized the health related quality of life (HRQOL) and the impact of pediatric disease in families. It included a study group (13 children who underwent ECMO) and a control group of 26 healthy children. The authors concluded that patients who have undergone ECMO support have lower HRQOL compared to the general pediatric population, but comparable to that of children with prevalent chronic diseases. ${ }^{21}$

This study has some limitations such as being a retrospective review in a single PICU, and the short follow-up period of the patients that underwent to ECMO in recent years. Another limitation is the use of an international database. Although it has the advantage of allowing benchmarking with other ECMO centers, we cannot control the variables that are registered. Nevertheless, this study has the strength of describing the largest cohort of neonatal and pediatric ECMO patients in Portugal.

During the last 10 years our work improved, as well as the technical skills, staff experience with the equipment and patient treatment. Since we implemented our ECMO program our results demonstrated that with a dedicated team and persistence we can have similar outcomes to those reported in the other neonatal and pediatric ECMO centers all over the world. We think that multicenter collaboration and uniformization of concepts and practices will reinforce the good results achieved so far.

\section{CONCLUSION}

This study represents our single-center experience over a 10-year period with the use of ECMO in neonatal and pediatric patients. We confirm that our PICU has now a wellestablished neonatal and pediatric ECMO program. This is a low volume ECMO center but has results that are not inferior to those reported in the ELSO registry.

The good results achieved reflect the organization of a multidisciplinary team and a continuous effort to improve our capabilities through the implementation of several learning programs, hands-on workshops and protocols for our staff (including consultants, doctors, residents and nurses).

\section{PROTECTION OF HUMANS AND ANIMALS}

The authors declare that the procedures were followed according to the regulations established by the Clinical Research and Ethics Committee and to the Helsinki Declaration of the World Medical Association, updated in 2013.

\section{DATA CONFIDENTIALITY}

The authors declare having followed the protocols in use at their working center regarding patients' data publication.

\section{COMPETING INTERESTS}

The authors have declared that no competing interests exist.

\section{FUNDING SOURCES}

This research received no specific grant from any funding agency in the public, commercial, or not-for-profit sectors. 


\section{REFERENCES}

1. Mosier J, Kelsey M, Raz Y, Gunnerson K, Meyer R, Hypes CD, et al. Extracorporeal membrane oxygenation (ECMO) for critically ill adults in the emergency department: History, current applications, and future directions. Crit Care. 2015;19:1-8.

2. Butt W, MacLaren G. Concepts from paediatric extracorporeal membrane oxygenation for adult intensivists. Ann Intensive Care 2016;6.

3. Bartlett R, Gazzaniga A, Toomasian J, Coran A, Roloff D, Rucker R. Extracorporeal membrane oxygenation (ECMO) in neonatal respiratory failure. 100 cases. Ann Surg. 1986;204:236-45.

4. Extracorporeal Life Support Organization. ECLS Registry Report Centers by year. 2020;2020:1-37. [accessed 2021 Feb 01]. Available from: https://www.elso.org/Portals/0/Files/Reports/2020_January/ International\%20Summary\%20January\%202020_page1.pdf.

5. Peek G, Mugford M, Tiruvoipati R, Wilson A, Allen E, Thalanany M, et al. Efficacy and economic assessment of conventional ventilatory support versus extracorporeal membrane oxygenation for severe adult respiratory failure (CESAR): a multicentre randomised controlled trial. Lancet. 2009;374:1351-63.

6. Bartlett R. Esperanza: The first neonatal ECMO patient. ASAIO J. 2017;63:832-43.

7. Marques R, Pereira S, Abecasis F, Valente S, Neves J, Abecasis M, et al. Uso de oxigenação extra corporal (ECMO) em recém-nascidos com hérnia diafragmática congénita - dois casos de sucesso. Acta Pediatr Port. 2013;44:172-5.

8. Rocha G, Soares P, Henriques-Coelho T, Correia-Pinto J, Monteiro J, Guimarães $\mathrm{H}$, et al. Neonatal extracorporeal membrane oxygenation: Initial experience of Hospital de São João. Rev Port Pneumol. 2014;20:336-40.

9. Vidmar I, Primožić J, Kalan G, Grosek S. Extracorporeal membranous oxygenation (ECMO) in neonates and children experiences of a multidisciplinary paediatric intensive care unit. Signa Vitae. 2008;3:S1721.

10. Makhoul M, Bitton-Worms K, Adler Z, Saeed A, Cohen O, Bolotin G. Extracorporeal Membrane Oxygenation (ECMO) - a lifesaving technology. Review and single-center experience. Rambam Maimonides Med J. 2019;10:e0013.

11. Burns S, Constantin N, Robles P. Understanding the long-term sequelae of ECMO survivors. Intensive Care Med. 2018;44:1144-7.
12. Bermudez C, Rocha R, Zaldonis D, Bhama J, Crespo M, Shigemura $\mathrm{N}$, et al. Extracorporeal membrane oxygenation as a bridge to lung transplant: Midterm outcomes. Ann Thorac Surg. 2011;92:1226-32.

13. Murphy D, Hockings L, Andrews R, Aubron C, Gardiner E, Pellegrino V, et al. Extracorporeal membrane oxygenation-hemostatic complications. Transfus Med Rev. 2015;29:90-101.

14. Hervey-Jumper SL, Annich G, Yancon A, Garton H, Muraszko K, Maher $C$, et al. Neurological complications of extracorporeal membrane oxygenation in children. J Neurosurg Pediatr. 2011;7:338-44.

15. Xie A, Lo P, Yan TD, Forrest P. Neurologic complications of extracorporeal membrane oxygenation: a review. J Cardiothorac Vasc Anesth. 2017;31:1836-46.

16. Extracorporeal Life Support Organization. ELSO Pediatric Respiratory Failure supplement to the ELSO general guidelines. 2013. [accessed 2021 Feb 01]. Available from: https://www.elso.org/Portals/0/IGD/ Archive/FileManager/6f129b235acusersshyerdocumentselsoguidelines forpediatricrespiratoryfailure1.3.pdf.

17. Cengiz P, Seidel K, Rycus P, Brogan T, Roberts J. Central nervous system complications during pediatric extracorporeal life support: Incidence and risk factors. Crit Care Med. 2005;33:2817-24.

18. Howard T, Kalish B, Rajagopal S, Williams K, Zalieckas J, Thiagarajan $\mathrm{RR}$, et al. Factors associated with mortality in children who successfully wean from extracorporeal membrane oxygenation. Pediatr Crit Care Med. 2018;19:875-83.

19. Alsoufi B, Al-Radi O, Gruenwald C, Lean L, Williams W, McCrindle B, et al. Extra-corporeal life support following cardiac surgery in children: analysis of risk factors and survival in a single institution. Eur $\mathrm{J}$ Cardiothoracic Surg. 2009;35:1004-11.

20. Merkle J, Azizov F, Sabashnikov A, Weixler V, Weber C, Djordjevic I, et al. Pediatric patients requiring extracorporeal membrane oxygenation in heart failure: 30-day outcomes; mid- and long-term survival. A single center experience. Artif Organs. 2019;43:966-75.

21. Varni J, Limbers C, Burwinkle T. Impaired health-related quality of life in children and adolescents with chronic conditions: a comparative analysis of 10 disease clusters and 33 disease categories/severities utilizing the PedsQL 4.0 Generic Core Scales. Health Qual Life Outcomes. 2007;5:43. 